UDC 327.56(6)+327(44)

Submitted: 03.12.2018

LBC 66.4(4Фpa),3

Accepted: 07.04.2019

\title{
FRANCE IN THE PROCESS OF THE RESOLUTION OF CONFLICTS ON THE AFRICAN CONTINENT
}

\author{
Olga A. Smirnova \\ National Research Lobatchevsky State University of Nizhny Novgorod, Nizhny Novgorod, Russian Federation
}

\begin{abstract}
Introduction. Historically, France has always had an impact on the African continent. Despite the independence of most French colonies in Africa, France managed to maintain its position in the region. The system called "Françafrique" was created. The first element of this system is economic cooperation, namely the fact, that Africa provides an opportunity to use its natural resources. Another important element is the military one. The White paper of France has repeatedly emphasized the strategic importance of Africa for the Fifth Republic. In the 2010s, the situation in the region deteriorated. France's assistance in overcoming internal crises in Mali, Chad, Libya can be considered an evidence of the increasing role of France in conflict resolution, and that is the thing which is analyzed by the author in this work. Methods and materials. While writing the article, both official documents of the French government and analytical works of famous French authors were used. Both general scientific methods (analysis, synthesis) and quantitative scientific methods (event analysis and content analysis) are used in the paper. Analysis. The article is devoted to the activities of France on the African continent in the period from 2011 to 2017. A Special attention is paid to the role of France in the conflict resolution of Africa. In particular, the paper considers France's support for the adoption of the UN resolution in response to the civil war in Libya in 2011 and military intervention in the country; military operations of the Fifth Republic in the Sahel zone Serval operation and in the Central African Republic - Sangaris operation, military presence of France in Djibouti. The paper emphasizes the key provisions of the French policy towards the African continent aimed at the preservation of French influence in the region. Results. In the article, the author comes to the following conclusions: France is not ready to abandon its military ambitions, it continues Barkhane operation, without stopping the fight against terrorism in the Sahel region; despite the competition from other countries (in particular, China and Japan), France's military presence in Eastern Africa remains the same, France does not intend to leave the base in Djibouti.

Key words: French military operations, intervention in Libya, French policy in Africa, Serval operation, Sangaris operation, Barkhane Operation.

Citation. Smirnova O.A. France in the Process of the Resolution of Conflicts on the African Continent. Vestnik Volgogradskogo gosudarstvennogo universiteta. Seriya 4. Istoriya. Regionovedenie. Mezhdunarodnye otnosheniya [Science Journal of Volgograd State University. History. Area Studies. International Relations], 2019, vol. 24, no. 3, pp. 147-157. (In French). DOI: https://doi.org/10.15688/jvolsu4.2019.3.13
\end{abstract}

УДК $327.56(6)+327(44)$

Дата поступления статьи: 03.12.2018

ББК 66.4(4Фра),3 Дата принятия статьи: 07.04.2019

\section{ФРАНЦИЯ В РАЗРЕШЕНИИ КОНФЛИКТОВ НА АФРИКАНСКОМ КОНТИНЕНТЕ}

\section{Ольга Анатольевна Смирнова}

Национальный исследовательский Нижегородский государственный университет им. Н.И. Лобачевского, г. Нижний Новгород, Российская Федерация

Аннотация. Исторически Франция всегда оказывала влияние на Африканский континент. Несмотря на обретение независимости большинством французских колоний в Африке, Франции удалось сохранить свои позиции в регионе. Появилась система под названием «Франсафрик». Первый ее элемент - экономическое сотрудничество, а именно предоставление Африкой возможности использования природных ресурсов. Дру- 
гой важный элемент - военный. В Белой книге Франции неоднократно подчеркивалась стратегическая важность Африки для Пятой республики. В 2010-е гг. ситуация в регионе обострилась. Помощь Франции в преодолении внутренних кризисов в Мали, Чаде, Ливии можно рассматривать как свидетельство возрастающей роли Франции в разрешении конфликтов, что и анализируется автором в данной работе. При написании статьи использовались как официальные документы французского правительства, так и аналитические работы известных французских авторов. В работе применяются как общенаучные методы (анализ, синтез), так и количественные (ивент-анализ и контент-анализ). Статья посвящена деятельности Франции на Африканском континенте в период с 2011 по 2017 год. Особое внимание уделяется роли Франции в разрешении конфликтов в Африке. В частности, рассматриваются поддержка Францией принятия резолюции ООН в ответ на гражданскую войну в Ливии 2011 г. и военная интервенция в данной стране; военные операции Пятой республики в зоне Сахеля (операция «Сервал») и в Центрально-Африканской Республике (операция «Сангарис»), военное присутствие Франции в Джибути. Выделяются ключевые положения французской политики по отношению к Африканскому континенту, направленные на сохранение французского влияния в регионе. В статье автор приходит к следующим выводам: Франция не готова оставить свои военные амбиции, она продолжает операцию «Бархан», не прекращая борьбу с терроризмом в регионе Сахеля; несмотря на конкуренцию со стороны других стран, в частности Китая и Японии, военное присутствие Франции на востоке Африки остается прежним, Франция не намерена покидать базу в Джибути.

Ключевые слова: военные операции Франции, интервенция в Ливии, политика Франции в Африке, операция «Сервал», операция «Сангарис», операция «Бархан».

Цитирование. Смирнова О. А. Франция в разрешении конфликтов на Африканском континенте // Вестник Волгоградского государственного университета. Серия 4, История. Регионоведение. Международные отношения. -2019. - Т. 24, № 3. - С. 147-157. - (На фр. яз.). - DOI: https://doi.org/10.15688/jvolsu4.2019.3.13

UDC 327.56(6)+327(44)

La réception à l'édition: 03.12 .2018

BBK 66.4(4Фpa),3

L'acceptation de l'article: 07.04.2019

\section{LA FRANCE DANS LA RÉSOLUTION DES CONFLITS SUR LE CONTINENT AFRICAIN}

\section{Olga Anatolievna Smirnova}

Université nationale de recherche Lobachevski de Nijni Novgorod, Nijni Novgorod, Russie

Résumé. Historiquement, la France influençait toujours le continent africain. Malgré l' indépendance obtenue par la majorité des ex-colonies françaises en Afrique, la France a réussi à garder ses positions dans cette région. Le système intitulé «Françafrique» a apparu. Son premier élément est la coopération économique, qui prévoit l'accès aux ressources naturelles. Un autre élément important est militaire. Le Livre blanc français a souligné à plusieurs reprises l'importance stratégique de l'Afrique pour la V-e République. Dans les années 2010 la situation dans la région s'est aggravée. Dans cet article l'auteur analyse l'aide de la France à la résolution des crises internes au Mali, au Tchad et en Libye. On a utilisé les documents officiels du gouvernement français, et des travaux analytiques des auteurs français. On utilise à la fois des méthodes scientifiques générales (analyse, synthèse) et quantitatives (event et content). L'article est consacré aux activités de la France en Afrique de 2011 à 2017. Une attention particulière est accordée au rôle de la France dans la résolution des conflits sur le continent africain. En particulier, le soutien de la France d'une résolution de l'ONU en réponse à la guerre civile en Libye en 2011 et l'intervention militaire dans ce pays; les opérations militaires de la V - ème République dans la zone sahélienne (opération Serval) et en République centrafricaine (opération Sangaris), la présence militaire française à Djibouti. On distingue les instruments clés de la politique française, visant à maintenir l'influence française dans cette région. Dans l'article l'auteur fait des conclusions suivantes: la France n'est pas prête à renoncer à ses ambitions militaires, elle poursuit l'opération Barkhan, la lutte contre le terrorisme dans la région du Sahel; malgré la concurrence d' autres pays, notamment la Chine et le Japon, la présence militaire de la France à l'est de l'Afrique reste la même, la France n'a pas l'intention de quitter la base à Djibouti.

Mots-clés: Opérations militaires françaises, intervention en Libye, politique française en Afrique, Opération Serval, Opération Sangaris, Opération Barkhan.

Citation. Smirnova O.A. La France dans la Résolution des Conflits sur le Continent Africain. Vestnik Volgogradskogo gosudarstvennogo universiteta. Seriya 4. Istoriya. Regionovedenie. Mezhdunarodnye otnosheniya [Science Journal of Volgograd State University. History. Area Studies. International Relations], 2019, vol. 24, no. 3, pp. 147-157. DOI: https://doi.org/10.15688/jvolsu4.2019.3.13 
Introduction. Historiquement, la France a toujours eu une énorme influence sur le continent africain. Malgré l'indépendance de la plupart des colonies françaises en Afrique, la France a réussi à maintenir sa position dans la région. Le Livre blanc sur la défense et la sécurité nationale définit l'Afrique comme une région stratégiquement importante pour la France [10]. Donc, il faut constater que la France a un intérêt sécuritaire et économique direct à la stabilité du continent africain [9].

En 2011, il y a eu le soi-disant « printemps arabe $»$ : un certain nombre de manifestations antigouvernementales au Moyen-Orient et en Afrique. Dans de nombreux pays, ces actions se sont transformées en affrontements avec les forces de l'ordre et, dans certains cas, se sont transformées en guerres civiles. Une situation similaire s'est produite en Libye, l'un des pays les plus développés de la zone sahélienne. Les manifestations contre la Jamahiriya se sont rapidement transformées en une guerre civile entre partisans et opposants aux activités du dirigeant du pays, Mouammar Kadhafi. L'aide apportée par la France pour surmonter les crises internes au Mali, au Tchad, en Libye peut être considérée comme une preuve du rôle croissant de la France dans la résolution des conflits, et c'est ce qui est analysé par l'auteur de cet ouvrage.

Méthodes. Lors de la rédaction de l'article, des documents officiels du gouvernement français et des travaux analytiques d'auteurs français célèbres ont été utilisés. L'article utilise à la fois des méthodes scientifiques générales (analyse, synthèse) et des méthodes scientifiques fréquentes (analyse des événements et analyse du contenu).

Analyse. Donc, les événements se sont développés si rapidement en Libye qu'il a fallu intervenir dans la situation émergente des acteurs internationaux. Le 26 février 2011, le Conseil de sécurité de l'ONU a adopté la résolution n ${ }^{\circ} 1970$ qui a adopté à l'unanimité une condamnationcontre le régime de Mouammar Kadhafi. Le 17 mars, avec le soutien actif de la France, la Résolution $\mathrm{n}^{\circ} 1973$ a été adoptée, qui est devenue la suite de la Résolution $n^{\circ} 1970$. La résolution a réaffirmé le ferme attachement de l'ONU à «la souveraineté, à l'indépendance, à l'intégrité territoriale et à l'unité nationale de la Jamahiriya arabe libyenne ». Aussi, elle permet aux pays qui le souhaitent de participer à une zone d'exclusion aérienne au-dessus de la Libye pour protéger la population civile, et de « prendre toutes mesures nécessaires, nonobstant le paragraphe 9 de la résolution 1970 (2011) pour protéger les populations et les zones civiles menacées d'attaque en Jamahiriya arabe libyenne » [22].

La France a soutenu le plus activement l'adoption de la résolution. Peu de temps avant, le 10 mars 2011, le Président de la République, Nicolas Sarkozy a reçu la délégation du « conseil national de transition ». Après cet entretien, Sarkozy a déclaré le statut officiel du conseil, de plus, cet organe est devenu un seul représentant de la Libye. 11 mars 2011, pendant le sommet de Bruxelles, la France et Royaume-Uni ont élabores une proposition d'instauration d'une zone d'exclusion aérienne. Mais, la chancelière allemande Angela Merkel a refusé cette idée, était soutenue par la majorité des états européens. Un tel événement a conduit au fait que Sarkozy s'est retrouvé isolé pendant le sommet G8 à Paris.

Cependant, la France était soudainement soutenue par la Ligue Arabe. L'organisation a proposée l'instauration des zones d'exclusion aérienne, aux mêmes conditions que la France et la Grande-Bretagneont élabores. Ce soutien de la Ligue Arabe a permisà Sarkozy et au ministre des Affaires étrangères Alain Juppé de commencer une bataille diplomatique dans le cadre du Conseil de Sécurité. Un nouvel développement a été reçu après le lancement des troupes gouvernementales en Benghazi, qui a provoqué un grand massacre. C'était évident que la création des zones d'exclusion aérienne est nécessaire. Les Etats-Unis ont finalement adoptés cette idée.

Le 19 mars, le sommet spécial à Paris a été tenu avec la participation des parties concernées. La Libye a prononcée les mesures pour conformer à cette résolution : un cessezle-feu et la création des zones d'exclusion aérienne. Mais malgré le respect supposé d'un cessez-le-feu, les attaques aériennes ont été continuées par le régime de Kadhafi, selon les représentants des rebelles [1]. Le même jour, le Président de la République a déclare le démarrage de l'opération militaire en Libye, avec son nom d'Harmattan : "d'ores et déjà, nos avions empêchent les attaques aériennes sur la ville de Benghazi » [5]. 
Malgré le fait que l'opération française était l'une des parties de l'Opération Unified Protector, la France a suivie ses propres motivations. Selon Sidney Blumenthal, conseiller et analyste du renseignement officieux de la Secrétaire d'État américaine, " les plans de Sarkozy étaient motivés par les questions suivantes :

- Le souhait d'obtenir une plus grande part de la production de pétrole en Libye ;

- Accroître l'influence française en Afrique du Nord ;

- Améliorer sa situation politique intérieure en France ;

- Fournir aux armées françaises l'occasion de réaffirmer leur position dans le monde ;

- Répondre à la préoccupation de ses conseillers sur les projets à long terme de Kadhafi de supplanter la France comme puissance dominante dans les pays africains francophones » [6].

En utilisant les principes de la théorie du réalisme politique et de la realpolitik, il faut dire que les quatre premiers points relevaient de l'intérêt national français, mais le dernier point était l'intérêt personnel du Président.

Pour effectuer l'opération Harmattan, la France a décidée de déployer les forces de l'Armée de l'air, de la Marine nationale et de l'Armée de terre, coopérant avec les forces de l'OTAN.

Cette opération a duré jusqu'au 21 octobre 2011. Ce jour-là, le ministre des Affaires étrangères Alain Juppé, a annoncé l'achèvement officiel de l'opération Harmattan. La veille, avec le soutien de l'armée de l'air française, Mouammar Kadhafi a été tué. En fait, c'était le but principal de l'opération, malgré le discours d'Alain Juppé, qui a déclaré le suivant: «L'ensemble du territoire libyen est sous le contrôle du CNT et sous réserve de quelques mesures transitoires, l'opération de l'Otan est arrivée à son terme. L'objectif qui était le nôtre, c'est-à-dire accompagner les forces du Conseil national de transition dans la libération de leur territoire, est maintenant atteint » [17].

$\mathrm{Au}$ fil du temps, les opinions sur l'opération ont changé. Depuis 7 ans, les opinions sont passées de sobres à fortement négatives. Cela est particulièrement évident dans l'intervalle de temps entre 2016 et 2018. Le rapport du Parlement britannique, publié le 14 septembre 2016, critique le déroulement de l'opération en Libye, reconnaissant le fait que, par exemple, « $\mathrm{La}$ stratégie du Royaume-Uni fut fondée sur des postulats erronés et sur une analyse partielle des preuves » [13]. De plus, " la Commission des Affaires étrangères a ainsi relevé plusieurs erreurs dans le processus décisionnel qui a amené Londres à intervenir militairement en 2011 avec la France » [13]. Ça signifie la révision des positions des membres de l'OTAN.

Cette année, le Président actuel de la République, Emmanuel Macron, a fortement critique l'intervention en Libye. Pendant son visite à Tunis, il a donné sa vision sur les évènements de 2011, en nommant l'intervention comme " une fausse idée », en précisant que «l'Europe, les Etats-Unis et quelques autres ont une responsabilité dans la situation actuelle en Libye », en ajoutant que « nous avons collectivement plongé la Libye, depuis ces années, dans l'anomie, sans pouvoir régler la situation » [3]. On peut supposer que cette critique n'est qu'une partie du programme politique de Macron. Cependant, il convient de noter qu'aucun des objectifs, selon le rapport Blumenthal, n'a été pleinement mis en œuvre à long terme : la France est encombrée par un autre acteur international majeur, la Chine, la production pétrolière n'est pas actuellement un sujet urgent pour le pays et l'opération n'a pas eu un impact positif sur la situation intérieure du pays, car elle n'a pas affecté la position de Sarkozy, qui a perdu aux élections de 2012 pour François Hollande.

Un an après la fin de l'opération en Libye, après l'inauguration de François Hollande, les priorités de la France en Afrique se sont quelque peu déplacées vers le sud et l'ouest de la région du Sahel. La France a continué à rester dans la région du Sahel, parlant d'un engagement à traiter les questions de préservation de la stabilité et de la sécurité régionales. À ce moment-là, dans certains pays de la région, la situation politique interne a commencé à se détériorer, en particulier au Mali et au Tchad. La question de l'éventuelle intervention militaire s'est ouverte après le rapport du célèbre diplomate français, ancien ministre des affaires étrangères, conseiller du gouvernement à l'époque de Mitterrand, Hubert Védrine. Dans son rapport, il a noté qu'une situation comme celle de la Libye est maintenant possible, la France devra intervenir. Il n'était pas clair seulement dans quel pays cela devrait se produire [23]. 
Au cours du novembre 2012, la situation a commencé à s'améliorer. Le 15 novembre 2012, François Hollande a reçu son homologue du Niger, Mahamadou Issoufou. Au cours des pourparlers, il s'est tourné vers les mines d'uranium situées à la frontière avec le Mali, qui éprouvait des difficultés à maintenir son indépendance, tombant dans la crise de l'État. Les deux pays ont exprimé leur préoccupation quant au manque de contrôle sur les mines. Ainsi, les prévisions de Védrine coïncidaient avec la réalité si rapidement que personne ne pouvait s'y attendre.

Le 11 janvier 2013, l'opération Serval a commencé. C'était l'opération militaire menée au Mali par l'armée française en vue de soutenir les troupes maliennes cherchant à repousser une offensive des groupes armés islamistes qui avaient pris le contrôle de la partie nord du pays, plus précisément, le territoire de l'Azawad [18]. François Hollande a aussi ajouté que les buts de l'Opération sont les suivants : « d'arrêter l'avancée des forces djihadistes en direction de Bamako, sécuriser la capitale du Mali et permettre au pays de recouvrer son intégrité territoriale » [21].

L'opération a commencé par des frappes aériennes sur les rebelles près de Sévaré. De même, comme dans l'opération Harmattan, l'Armée de l'Air française, la Marine et l'Armée de terre française ont participées à la conduite des opérations militaires. Dans un premier temps, l'armée française-malienne commune a fait face à la résistance des rebelles, qui ont pu combattre l'attaque initiale. Cependant, dans quelques semaines, l'armée conjointe (en partie, grâce au soutien de ceux engagés dans l'opération Épervierau Tchad de l'armée de l'air) a pu se rapprocher beaucoup de Bamako en capturant des objets aussi importants que Gao, Tombouctou et Kidal. Ainsi que la boucle du Niger, stratégiquement importante.

Le 25 avril 2013, la Mission multidimensionnelle intégrée des Nations unies pour la stabilisation au Mali (MINUSMA) a été créée par la Résolution 2100 du Conseil de Sécurité de l'ONU. À partir de ce moment-là, l'opération Serval a commencé à se composer d'un grand nombre de petites opérations menées conjointement avec les forces des Nations Unies : Netero, Centaure, Dragon, Constrictor et Hydre. À partir de ce moment, le vecteur général de l'opération a également changé: les petites opérations ont commencé à être ponctuelles et les opérations contre les entités terroristes sont devenues plus fréquentes.

En 2014, il y a eu une réduction de la présence française au Mali. Le 8 janvier, François Hollande a annoncé la réduction du contingent de soldats à 1600 des 2500 existants. De plus, en un mois il fallait réduire le nombre de 600 autres personnes. Le commandement français a estimé qu'il suffirait de 1000 personnes pour finir l'opération, dont « l'essentiel de la mission a été accompli » [16].

Malgré la fin prochaine de l'opération, en mai, les forces alliées ont survécu à la reprise des affrontements dans le nord du Mali, conséquence du meurtre du légionnaire français. Peu de temps après, il a été décidé d'augmenter la présence de troupes, retardant ainsi l'achèvement possible de l'opération Serval pour une durée indéterminée.

Finalement, le 13 juillet 2014, le ministre français de la Défense, Jean-Yves Le Drian, a annoncé des changements dans l'opération militaire dans la région du Sahel. L'opération Serval est terminée, elle est déclarée réussie, et aussi elle se transforme en une opération plus globale Barkhane, dont le but est annoncé comme la lutte « contre le terrorisme dans l'ensemble du Sahel » [2].

L'opération Serval a été reconnue comme réussie, ses objectifs, à savoir, la suppression des opposants aux autorités actuelles et la stabilisation de la situation politique au Mali, ont été réalisés. Cependant, cette opération n'est pas devenue bénéfique pour la France, le pays n'a pas pu en tirer les profits nécessaires, néanmoins, ayant renforcé ses positions dans la région du Sahel.

Le 1er août 2014, une nouvelle opération a été lancée, nom de code Barkhane. Elle visait à développer les succès des précédentes opérations Serval et Épervier, mais l'ampleur de l'exercice s'est considérablement élargie: les cinq pays formant le G5: la Mauritanie, le Mali, le Burkina Faso, le Niger et le Tchad sont devenus une vaste zone d'opération antiterroriste française, qui a été rejoint par environ 3000 soldats.

Les principaux ennemis des alliés pendant l'opération de Barkhane sont les djihadistes de divers groupes. Leur nombre ne se prête pas à un calcul précis: selon les estimations préliminaires, leur nombre est de 300 à 3000 [15].

$\mathrm{Au}$ début de l'opération c'était nécessaire de résoudre la question fondamentale : si de faire 
une nouvelle intervention militaire en Libye ou non. A cette époque, sa partie sud-ouest était la base pour un grand nombre de terroristes. En France, le gouvernement y avait une scission en 2 parties: le premier, représenté par le ministère de la Défense, a estimé la nécessité d' " agir » en Libye, afin d'éviter une nouvelle détérioration de la situation dans le pays, tandis que d'autres, au nom du ministère des Affaires étrangères était strictement opposé à l'idée d'une invasion du pays, en insistant sur une solution politique au problème. François Hollande, à la fin, a pris le parti de la diplomatie française. Jean-Yves Le Drian est resté d'accord avec ce choix [8].

$\mathrm{Au}$ cours de l'opération, qui se poursuit encore aujourd'hui, de nombreuses opérations militaires ont été menées pour lutter contre les groupes armés opérant dans les pays du Sahel. Au départ, la lutte a été menée avec des partisans d'AQMI (Al-Qaïda au Maghreb islamique). Environ un an et demi de nombreuses batailles entre les forces alliées et les rebelles de l'organisation terroriste ont eu lieu. Après l'élimination du chef de l'AQMI, et la reconnaissance finale de la défaite de cette organisation, la lutte a commencé à être menée contre des formations plus petites dispersées sur tout le territoire du G5. Cependant, depuis décembre dernier, les plus grands rivaux des alliés sont devenus les plus grandes organisations djihadistes en Afrique, comme Boko Haram et l'État islamique dans le Grand Sahara. La résistance est également rendue par le Groupe de soutien à l'islam et aux musulmans, proches de l'EI. Les troupes alliées mènent des raids aériens réguliers sur des territoires appartenant à des terroristes. Cependant, l'opération n'est pas dans sa phase finale.

Parlant de la présence militaire de la France dans la région du Sahel, il est à noter qu'en organisant l'opération contreterroriste militaire Barkhane, la France résout deux problèmes :

1) soutient la stabilité et la sécurité régionales, agissant en tant que le garant et le principal défenseur des pays s'opposant aux activités des groupes terroristes armés;

2) maintient sa présence dans la région, qui a été remise en question immédiatement après la fin de la période coloniale du pays. L'alliance dite Françafrique, qui est en grande partie de nature socio-économique, est soutenue en partie par la composante militaro-politique.
Le Sahel reste la zone la plus active des opérations militaires, à laquelle la Cinquième République est impliquée. Malgré les protestations de l'opposition, le gouvernement actuel n'a pas l'intention de réduire le contingent de soldats dans le G5, car on pense que cette voie assure non seulement la sécurité des alliés africains, mais aussi la sécurité de la France.

La France, après l'effondrement du système colonial, a conservé son intérêt non seulement dans les colonies du nord et du nord-ouest de l'Afrique, mais aussi dans la partie centrale du continent. C'est la République centrafricaine, qui est également devenue le site d'une opération militaire menée avec le soutien de l'armée française.

La République centrafricaine a régulièrement demandé l'assistance de la France pour résoudre les contradictions politiques internes. L'opération Sangaris était la septième intervention sanctionnée par le gouvernement du pays africain. Le contexte de cette opération a été les événements de l'année 2012 : le président du pays, François Bozizé, s'est tourné vers la France et les Etats-Unis pour faire face à la montée de l'agression due aux activités de l'organisation musulmane Séléka [12]. Cette organisation est connue pour ses méthodes agressives de combat, donc elle était attestée aux rebelles. Séléka s'oppose en particulier à la majorité catholique de la République centrafricaine. La situation s'est aggravée juste après les élections présidentielles. Un candidat pro-musulman, Michel Djotodia a gagné, ce qui a affecté la situation politique intérieure. Les provocations ont commencé entre les catholiques et les musulmans, qui se sont transformés en affrontements armés, les troubles ont conduit au fait que d'ici octobre 2013, la république était incontrôlable, le pays se trouvait au bord du chaos [4].

Il y avait une menace de génocide, que la France a déclaré. A bien des égards, c'est la Ve République qui est devenue le principal sponsor de la Résolution 2127, qui a conduit à la création Mission internationale de soutien à la Centrafrique sous conduite africaine (MISCA). La mission a été lancée officiellement pour mettre fin à la « faillite totale de l'ordre public, l'absence de l'état de droit et les tensions interconfessionnelles » [14]. Mais le fait plus important est que la Résolution a officiellement autorisée l'intervention militaire française. 
Après 11 jours de formation préliminaire et déploiement de troupes, et le déploiement du dispositif militaire, dont une partie est restée de l'opération précédente, Boali, l'armée française était prête à commencer l'opération Sangaris.

Conditionnellement, cette opération peut être divisée en 4 stades: le début et le désarmement primaire des groupes armés, l'établissement d'un niveau minimum de sécurité et la croissance de la présence de la MISCA, l'installation de la MISCA et le déploiement de force de réaction rapide.

Au cours du premier mois de l'armée française, il réussit à ébranler de manière significative les positions de Séléka, tuant le représentant du haut commandement de l'organisation et procédant au désarmement primaire des représentants individuels des insurgés.

Depuis janvier 2014, le partenariat entre le commandement français et le commandement de l'opération onusienne se développe, des opérations conjointes sont menées. En deux semaines, les alliés ont été en mesure de réduire considérablement l'activité de la Séléka et de mener un autre désarmement. Cependant, la situation change lorsque les forces locales d'autodéfense sous le nom d'anti-balaka sont divisées en deux parties: modérée et radicale. $\mathrm{Si}$ les modérés étaient prêts à déposer les armes et à sortir pacifiquement du conflit, les radicaux étaient déterminés à attaquer la population musulmane pacifique.

Depuis mars 2014, le principal opposant des forces conjointes de la France et des Nations Unies sont des radicaux anti-balaka. Les attaques de Séléka ont continué. La situation menaçait de devenir incontrôlable. Cependant, depuis juin 2015, après le regroupement des troupes françaises et le déploiement de forces de réaction rapide, la situation a radicalement changé. Les forces rebelles sont devenues plus faibles.

Le 30 mars 2016 était l'inauguration du nouveau président de la République centrafricaine, Faustin-Archange Touadéra. Cela signifiait pour la France non seulement la fin de la période de transition, mais aussi la réussite de l'opération ellemême. Jean-Yves Le Drian, présent lors de l'inauguration, a annoncé que l'opération Sangaris serait achevée pendant 2016 [19]. Enfin, la visite de François Hollande a Bangui le 13 mai 2016 a fini l'opération Sangaris, qui a été officiellement proclame fermée.

L'Opération Sangaris, malgré la critique des observateurs internationaux et une certaine complexité dans la conduite a été couronnée de succès pour la France. La paix a été restauré, de plus, la période de transition a été conclue par la sélection du président, proche a la France. En outre, cette opération a souligné l'importance de la France pour la République centrafricaine. La France a confirmé son droit de transmettre son influence sur le pays, qui, comme les pays du G5 du Sahel, s'inscrit s'intègre dans le projet de Françafrique.

Chaque pays revendiquant le statut de grande puissance possède des bases militaires étrangères : la Russie, par exemple, a des bases en Arménie et au Kirghizistan, en Biélorussie et au Kazakhstan, au Vietnam et en Syrie, le Royaume-Uni continue de posséder des bases à Chypre, possède des bases au Canada, à Bahreïn, au Kenya, les États-Unis ont un grand nombre de bases militaires partout dans le monde. La France essaie de suivre les collègues des pays du G5 du Conseil de sécurité de l'ONU, ainsi que ceux qui n'y sont pas inclus, mais prétendent être une grande puissance. Dans le contexte de la présence militaire française en Afrique, l'objet le plus notable est la base étrangère de la France dans un petit Etat de l'est du continent, Djibouti.

Sous le nom « les forces françaises stationnées à Djibouti ", ce contingent français est plus nombreux et plus important hors Métropole. En 1977, Djibouti a reçu son indépendance. Malgré ce fait, les deux gouvernements ont signés le protocole provisoire. Ce protocole a fixé le règlement du stationnement des troupes françaises. Il aussi confirme la coopération militaire des deux pays [7]. L'accord de la coopération militaire a été prolongé plusieurs fois. Maintenant, le Traité de coopération en matière de défense signé le 21 décembre 2011 et entré en vigueur le 1 mai 2014 est le document qui détermine les relations entre la France et la République de Djibouti dans le cadre des affaires militaires.

Malgré la présence massive des armées françaises au territoire de la République de 
Djibouti, le nombre des militaires diminue chaque année. Maintenant, il y a 1450 militaires déployés, mais cette base reste la première base hors Métropole, en parlant du nombre des militaires.

Selon le site du Ministere des Armees, la base djiboutienne s'articule autour de 5 emprises principales :

- le 5e régiment interarmes d'outre-mer (5e RIAOM) ;

- le détachement de l'aviation légère de l'armée de terre (DETALAT) armant 2 hélicoptères Puma et 1 Gazelle (en 2014, la base a reçu 2 hélicoptères Puma et 1 Gazelle);

- la base aérienne 188 (BA 188) avec ses 4 avions de défense aérienne Mirage 2000-5, 1 avion de transport tactique C160 Transall, 2 hélicoptères Puma ;

- la base navale ;

- le centre d'entraînement au combat et d'aguerrissement au désert de Djibouti (CECAD) [11].

En ce qui concerne le 5e regiment interarmes d'outre-mer, il faut souligner que c'est le dernier régiment interarmes. En étant le régiment de tradition de Djibouti, il possede une posture opérationnelle permanente.

En parlant de la base navale, il faut dire que la Marine nationale possède en Djibouti des forces spéciales, utilisées dans les cas urgents, par exemple, dans la lutte contre la piraterie autour de la Corne de l'Afrique.

La base en Djibouti est aussi appelée comme la base opérationnelle avancée, parce qu'elle est une plateforme stratégique, opérationnelle et logistique pour la France.

En ce qui concerne les missions de la base, le ministère des Armées a proclamé que les forces françaises stationnées à Djibouti :

- sont en mesure de soutenir les opérations dans la zone en facilitant notamment les mouvements des forces (acheminements, désengagements, relèves humaines et matérielles);

- fournissent un point d'appui logistique aéroportuaire aux forces françaises mais aussi aux forces djiboutiennes et aux contingents alliés engagées dans la région;

- forment un réservoir de forces aguerries, acclimatées et entraînées rapidement projetables en cas de crise dans la sous-région;

- assurent une présence militaire en mesure d'assurer la protection des ressortissants dans leur zone de responsabilité permanente (ZRP).

Les autres critères de la mission des FFDj consistent à :

- mettre en œuvre la coopération militaire bilatérale avec la République de Djibouti en contribuant à la défense du territoire djiboutien, de son espace aérien et de ses eaux territoriales et en se tenant prêtes à fournir, si besoin, un appui aux opérations de secours sur terre et en mer ;

- animer la coopération régionale avec les alliés présents dans la sous-région et soutenir, le cas échéant, les opérations multinationales comme l'opération européenne de lutte contre la piraterie, Atalante. A ce titre, les FFDj accueillent les détachements espagnols et allemand et le FHQSA (état-major de soutien logistique) et assurent le soutien des bâtiments français mais aussi étrangers engagés dans cette opération ;

- accompagner les Etats africains de leur ZRP dans l'appropriation de leur sécurité nationale collective. Pour cela, les FFDj délivrent des formations et contribuant à la préparation opérationnelle des contingents africain avant leur engagement au sein de missions multinationales ou d'opérations de maintien de la paix. A ce titre, depuis 2006, les FFDj envoient un détachement d'instruction opérationnelle (DIO) pour la formation des bataillons ougandais aux missions de maintien de la paix et destinés à être déployés en Somalie dans le cadre de l'opération AMISOM ;

- réaliser des actions civilo-militaires au profit de la population régionale; contribuer à l'aguerrissement en milieu désertique des unités permanentes ou en mission de courte durée des FFDj mais aussi des unités ou des écoles venues de métropole, des forces djiboutiennes ou encore des forces alliées grâce aux infrastructures du CECAD [11].

Certaines opérations africaines ont été menées avec le soutien de cette base, comme les opérations Boali, Sangaris en Centrafrique et Barkhane au Sahel.

Maintenant, Djibouti est devenu la base pour d'autres pays qui ont une grande capacité militaire, le Japon a sa base ici, aussi l'occasion de sa propre base est utilisée par l'Italie et les États-Unis. Cependant, cela n'empêche pas la France de 
rester dans une position privilégiée, ayant plus d'influence sur ce pays africain que d'autres acteurs possédant des bases militaires. Pour la France, un tel arrangement est stratégiquement important, puisque leur domaine d'intérêt en Afrique n'est pas si éloigné de Djibouti, ce qui laisse une grande opportunité pour la manœuvre la plus rapide.

Conclusion. Résumant tout ce qui précède, il est nécessaire de mettre en évidence les principales dispositions relatives a la participation de la France dans la résolution des conflits en Afrique et a l'influence de la France sur le Continent :

- la France utilise ses possibilités militaires comme l'un des instruments de sa politique étrangère ;

- malgré la baisse notable de son rôle sur le continent, la France reste un acteur fort dans certains zones africaines, notamment, Sahel et l'Est de l'Afrique ;

- la France n'est pas prête de laisser ses ambitions militaires, mettant les relations socioéconomiques au premier plan, elle continue l'opération Barkhane, ne cessant pas la lutte contre le terrorisme dans une région du Sahel ; le discours d'Emmanuel Macron à Ouagadougou semble symbolique, parce qu'elle prononce : « Il n'y a plus de politique africaine de la France » [20], mais ça ne signifie pas que les rapports socioéconomiques entre la France et l'Afrique vont être dominant, la France a besoin de son déplacement stratégique en Afrique ;

- malgré la concurrence forte avec les autres pays du monde (notamment, la Chine et le Japon), la présence militaire du pays à l'Est du continent reste le même, la France n'est pas prête de quitter la base en Djibouti ;

- la France a déjà entrée dans la période de repenser ce qui s'était passé auparavant; maintenant, l'opération Harmattan est souvent considérée comme une erreur : « La France n'a pas participé à la guerre en Irak et elle a eu raison. Et elle a eu tort de faire la guerre de cette manière en Libye » [24];

- la France reste le pays avec l'influence large en Afrique, mais pour avoir les relations harmonieuses, il faut trouver un équilibre entre « la France comme le grand frère » et « La France comme le partenaire, l'ami, le camarade ».

\section{REFERENCES}

1. Abbas M., MacSwan A. Les forces de Mouammar Kadhafi dans Benghazi. Le Point. 2011. Mars le 19. URL: http://www.lepoint.fr/fil-info-reuters/ les-forces-de-mouammar-kadhafi-dans-benghazi-1903-2011-1308632_240.php (date d'accès : 03.03.2018).

2. Au Mali, l'opération « Serval » est « de fait terminée». Le Monde. 2014. Juillet le 13. URL: http:// www.lemonde.fr/afrique/article/2014/07/13/1-operationserval-remplacee-par-une-operation-antiterroriste 4456261_3212.html (date d'accès : 03.03.2018).

3. Bobin F., Pietralunga C. A Tunis, Macron critique l'intervention militaire de l'OTAN en Libye en 2011. Le Monde. 2018. Février, le 1. URL : http:// www.lemonde.fr/afrique/article/2018/02/01/a-tunisemmanuel-macron-critique-l-intervention-militaire-de1-otan-en-libye-de-2011_5250601_3212.html (date d'accès : 03.03.2018).

4. Châtelot $\mathrm{C}$. La Centrafrique à l'heure du chaos. Le Monde. 2013. Septembre, le 25. URL : http:// www.lemonde.fr/afrique/article/2013/09/25/lacentrafrique-a-1-heure-du-chaos_3484325_3212.html (date d'accès : 03.03.2018).

5. Clarisse Y. Sarkozy annonce que l'opération militaire a commencé en Libye. Reuters. 2011. Mars, le 19. URL : http://fr.reuters.com/article/topNews/ idFRPAE72I0AM20110319 (date d'accès : 03.03.2018).

6. d'Alançon F. Intervention en Libye: les motivations de Sarkozy. La Croix. 2016. Septembre le 15. URL : http://monde.blogs.la-croix.com/ intervention-en-libye-les-motivations-de-sarkozy/ 2016/09/15/ (date d'accès : 03.03.2018).

7. Décret $n^{\circ} 85-1171$ du 5 novembre 1985. P. : Journal Officiel, 1985. URL : https://www.legifrance. gouv.fr/jo_pdf.do?numJO $=0 \&$ date $J O=19851110$ \&numTexte $=\&$ pageDebut $=13060 /$ (date d'accès : 02.03.2018).

8. Dumas M. Bruits d'intervention militaire en Libye. Orient XXI. 2015. Janvier, le 6. URL : http:// orientxxi.info/magazine/bruits-d-intervention-militaireen,0781 (date d'accès : 03.03.2018).

9. La revue stratégique de défense et de sécurité nationale 2017. P. : Direction générale des relations internationales et de la stratégie, 2017. URL : https:// www.defense.gouv.fr/content/download/514684/ 8664656/file/2017-RS-def1018.pdf (date d'accès : 02.03.2018).

10. Le livre blanc sur la défense et la sécurité nationale.. . : Direction de l'information légale et administrative, 2013. URL : https://www.defense. gouv.fr/content/download/206186/2286591/file/Livreblanc-sur-la-Defense-et-la-Securite-nationale $\%$ 202013.pdf(date d'accès: 02.03.2018).

11. Les forces françaises stationnées à Djibouti. URL : https://www.defense.gouv.fr/ema/forces- 
prepositionnees/djibouti/dossier/les-forces-francaisesstationnees-a-djibouti (date d'accès: 02.03.2018).

12. Le président centrafricain appelle la France et les Etats-Unis à l'aide. La Libération, 2012. Décembre, le 27. URL : http://www.liberation.fr/planete/2012/12/ 27/le-president-centrafricain-appelle-la-france-et-lesetats-unis-a-l-aide_870245 (date d'accès : 04.03.2018).

13. Libye : l'offensive anti-Kadhafi de 2011 basée sur « des postulats erronés ». France 24. 2016. Septembre, le 14. URL : http://www.france24.com/fr/ 20160914-intervention-militaire-2011-libye-rapportparlementaires-britanniques-cameron-sarkozy (date d'accès : 04.03.2018).

14. L'ONU autorise une intervention française en Centrafrique. $t d g . c h$. 2013. Décembre, le 5. URL: https:// www.tdg.ch/monde/afrique/L-ONU-autorise-uneintervention-francaise-en-Centrafrique/story/17162258 (date d'accès : 04.03.2018).

15. Mali: l'armée française remet la pression sur les jihadistes dans le Nord. fr.news.yahoo.com. 2016. Juillet, le 12. URL : https://fr.news.yahoo.com/malilarmee-francaise-remet-pression-jihadistes-nord124707939.html (date d'accès : 04.03.2018).

16. Mali: la force française va être ramenée progressivement à 1000 hommes. L'Express.fr. 2014. Janvier, le 8. URL : https://www.lexpress.fr/actualite/ monde/afrique/mali-la-force-francaise-va-etreramenee-progressivement-a-1000-hommes 1312549.html (date d'accès : 04.03.2018).

17. Merchet J-D. Libye : « l'opération militaire est terminée », déclare Alain Juppé ». Marianne.net. 2011. Mars, le 24. URL : http://www.marianne.net/ blogsecretdefense/Libye-1-operation-militaire-estterminee_-declare-Alain-Juppe_a411.html (date d'accès : 04.03.2018).

18. Mission Multidimensionnelle Intégrée des Nations Unies pour la Stabilisation au Mali, le HautCommissariat des Nations unies aux droits de l'homme. Rapport conjoint sur les violations des droits de l'homme et $d u$ droit international humanitaire commis lors des événements de Kidal, les 16, 17 et 21 mai 2014. URL : http://www.ohchr.org/ Documents/Countries/ML/RapportKidal_Dec2015. pdf(date d'accès : 03.03.2018).

19. Point de situation des opérations du 31 mars 2016. URL : https://www.defense.gouv.fr/operations/ operations/centrafrique/actualites/point-de-situation-desoperations-du-31-mars-2016 (date d'accès: 03.03.2018).

20. Pour Macron, «il n'y a plus de politique africaine de la France». L'Indépendant. 2017. Novembre, le 28. URL : www.lindependant.fr/2017/11/ 28/pour-macron-il-n-y-a-plus-de-politique-africainede-la-france,3074397.php (date d'accès : 04.03.2018).

21. SLM Gary. Et si la France créait le désordre pour pérenniser sa présence! Afrique sur 7. 2016. Octobre, le 21. URL : https://www.afrique-sur7.fr/ 372950-mali-et-si-la-france-creait-le-desordre-pourperenniser-sa-presence (date d'accès : 04.03.2018).

22. Texte du projet de la Résolution 1973 adoptée le 17 mars 2011. URL : http://www.un.org/fr/ documents/view_doc.asp?symbol=S/RES/1973(2011) (date d'accès: 03.03.2018).

23. Védrine H. Rapport pour le Président de la République Française sur les conséquences du retour de la France dans le commandement intégré de l'OTAN, sur l'avenir de la relation transatlantique et les perspectives de l'Europe de la défense, 2012, novembre le 14. URL : diplomatie.gouv.fr/fr/IMG/pdf/ Rapport_H_VEDRINE_V7_-_definitive_cle05be84. pdf(date $d$ 'accès : 03.03.2018).

24. Werty R. Emmanuel Macron: « L'Europe n'est pas un supermarché. L'Europe est un destin commun ». Letemps.ch. 2017. Juin, le 21. URL : https://www. letemps.ch/monde/emmanuel-macron-leurope-nestun-supermarche-leurope-un-destin-commun (date d'accès : 04.03.2018).

\section{Information about the Author}

Olga A. Smirnova, Candidate of Sciences (Politics), Director of the French Center, Associate Professor, Department of Foreign Languages and Linguistics, National Research Lobachevsky State University of Nizhny Novgorod, Ulyanova St., 2, 603005 Nizhny Novgorod, Russian Federation, oasmirnova64@mail.ru, https://orcid.org/0000-0003-3822-2154

\section{Информация об авторе}

Ольга Анатольевна Смирнова, кандидат политических наук, директор Французского центра, доцент кафедры иностранных языков и лингвокультурологии, Национальный исследовательский Нижегородский государственный университет им. Н.И. Лобачевского, ул. Ульянова, 2, 603005 г. Нижний Новгород, Российская Федерация, oasmirnova64@mail.ru, https://orcid.org/0000-0003-3822-2154 


\section{Information de l'auteur}

Olga Anatolievna Smirnova, candidat ès sciences politiques, Directrice du Centre français, maître de conférences du Département des langues étrangères et de la linguistique culturelle, Université nationale de recherche Lobachevski de Nijni Novgorod, 2 rue Oulyanova, 603005 Nijni Novgorod, Russie, oasmirnova64@mail.ru, https://orcid.org/0000-0003-3822-2154 